\title{
The Relationship between Plasma Erythropoietin Levels and Symptoms of Attention Deficit Hyperactivity Disorder
}

\author{
Se-hoon Shim ${ }^{1, *}$, Yong-Ku Kim ${ }^{2, *}$, Young Hwangbo ${ }^{3}$, Hee-jung Yoon ${ }^{4}$, Ji Sun Kim', Youn Jung Lee, \\ Young Sup Woo $^{6}$, Won-Myong Bahk \\ ${ }^{1}$ Department of Psychiatry, Soonchunhyang University Cheonan Hospital, Soonchunhyang University College of Medicine, Cheonan, ${ }^{2}$ Department \\ of Psychiatry, Korea University Ansan Hospital, Ansan, ${ }^{3}$ Department of Preventive Medicine, Soonchunhyang University College of Medicine, \\ Cheonan, ${ }^{4}$ Korean Society of Infectious Diseases, Seoul, ${ }^{5}$ Department of Psychiatry, Gyeongsang National University School of Medicine and \\ Gyeongsang National University Hospital, Jinju, ${ }^{6}$ Department of Psychiatry, College of Medicine, The Catholic University of Korea, Seoul, Korea
}

Objective: There are animal models associating dopamine dysfunction with behavioral impairments that model attention deficit hyperactivity disorder (ADHD). Erythropoietin (EPO) has trophic effects on dopaminergic neurons. The aim of this study was to examine the EPO plasma levels and determine whether there was any correlation between plasma EPO levels and clinical characteristics of ADHD.

Methods: Plasma EPO levels were measured in 78 drug-naïve children with ADHD and in 81 healthy children. The severity of ADHD symptoms was determined by scores on the Korean ADHD Rating Scale (K-ARS) in ADHD children and healthy controls.

Results: The difference between median plasma EPO levels in ADHD children and in healthy controls was not statistically significant. Adjusting for age and sex, a linear regression analysis showed that inattention score was significantly higher in the second highest tertile of plasma EPO compared to those in the lowest tertile. Hyperactivity-impulsivity score was significantly higher in the highest tertile of plasma EPO compared to those in the lowest tertile. Moreover, total K-ARS scores were significantly higher in the second highest tertile of plasma EPO compared to those in the lowest tertile.

Conclusion: These findings suggest that plasma EPO levels were related to some ADHD symptoms, which could be used in the monitoring of the disorder.

KEY WORDS: Attention deficit hyperactivity disorder; Erythropoietin; Neuroprotection; Neurodevelopment.

\section{INTRODUCTION}

Attention deficit hyperactivity disorder (ADHD) is a neurodevelopmental disorder [1] that involves an apparent delay in the development of impulse control. Characteristics of ADHD include inattention, impulsivity, and hyperactivity that are long-lasting and atypical compared with people at similar developmental levels. ADHD has a worldwide prevalence of 5.3\% [2]. In Korea, ADHD has a

Received: July 31, 2020 / Accepted: August 16, 2020

Address for correspondence: Won-Myong Bahk

Department of Psychiatry, Yeouido St. Mary's Hospital, College of Medicine, The Catholic University of Korea, 10 63-ro,

Yeongdeungpo-gu, Seoul 07345, Korea

E-mail:wmbahk@catholic.ac.kr

ORCID: https://orcid.org/0000-0002-0156-2510

*These authors contributed equally to this study as co-first authors. prevalence of $4.5 \%$ [3]. ADHD is a common neuropsychiatric disorder in children and adolescents [4]. The underlying pathophysiology of ADHD could involve dysregulation of the noradrenergic fronto-cortical inhibition of dopaminergic striatal structures [5].

Erythropoietin (EPO) is a hematopoietic factor that controls the survival and proliferation of bone marrow progenitor cells during erythropoiesis [6]. In addition to the kidneys, the liver is an important source of EPO, and the hepatocytes and Ito cells are primarily responsible for EPO synthesis in the liver $[7,8]$. A variety of organs, such as the placenta, testis, lung, spleen, and brain have been shown to express EPO $[9,10]$. Cultured astrocytes have been shown to produce immunoreactive EPO $[11,12]$. There is accumulating evidence supporting the fact that EPO has significant neuroprotective potential in disorders

(c) This is an Open-Access article distributed under the terms of the Creative Commons Attribution Non-Commercial License (http://creativecommons.org/licenses/by-nc/4.0) which permits unrestricted non-commercial use, distribution, and reproduction in any medium, provided the original work is properly cited. 
of the peripheral nervous system [13]. EPO effects include direct neurotrophic effects with EPO-mediated neuroprotection mechanisms [14]. High plasma levels of endogenous EPO were associated with protection from acute neurologic deficits in Kenyan children with cerebral malaria (CM). This result indicates a neuroprotective role of EPO in CM [15].

There are a few animal models relating dopamine dysfunction with behavioral impairments that model ADHD [16]. EPO, the important modulator of erythroid progenitor cells has trophic effects on dopaminergic neurons, and promotes neuronal survival as well. EPO activates the CREB transcription pathway and increases brain-derived neurotrophic factor (BDNF) expression and production, which contributes to erythropoietin-mediated neuroprotection [17]. There is a high plasma BDNF level in untreated ADHD patients, and high plasma BDNF levels were significantly associated with the severity of inattention symptoms [18]. In addition, the total serum hepcidin levels in ADHD patients may cause iron dysregulation in ADHD patients [19].

However, to the best of our knowledge, no previous report has investigated the association between ADHD and EPO. Therefore, the aim of the present study was to examine the EPO plasma levels and determine whether there was any correlation between plasma EPO levels and clinical characteristics of ADHD.

\section{METHODS}

\section{Participants}

In total, 78 children with ADHD and 81 healthy children were enrolled. Children with ADHD were recruited from the outpatient and inpatient clinics of the Soonchunhyang University Cheonan Hospital. They were medication-naïve. Individuals with a full-scale IQ lower than 70, neurological disorders, seizure disorders, pervasive developmental disorders, Tourette's disorder or chronic tic disorder, bipolar mood disorders, or psychotic disorders were excluded. We recruited control-group participants from one elementary school in Cheonan after excluding children according to the following criteria: 1) having major medical/neurological/psychiatric/developmental diseases, as determined through parent questionnaires; 2) exhibiting serious behavioral problems, as determined through teacher questionnaires; or 3) presenting a total Korean version of the ADHD Rating Scale (K-ARS) score falling above the 90th percentile cut-off point.

The study protocol was approved by the Institutional Review Board of the Soonchunhyang University Cheonan Hospital (no. 2017-11-020-001). Parents/guardians provided written informed consent, and the children or adolescents provided verbal assent regarding participation in this study.

\section{Measures}

\section{Assessment instruments}

Diagnostic assessments of psychiatric disorders in the children with ADHD were made according to the Diagnostic and Statistical Manual of Mental Disorders 4th edition criteria, the Kiddie-Schedule for Affective Disordersand Schizophrenia for School-Age Children-Present and Lifetime-Korean Version (KSADS-PL-K) [20]. The K-SADS-PL-K interviews with all children with $\mathrm{ADHD}$ and either one or both parents of each child were carried out by clinically experienced child/adolescent psychiatrists. All children with ADHD completed a comprehensive and standardized battery of psychological tests, including the Korean Educational Development Institute-Wechsler Intelligence Scale for Children [21] and the ADHD Rating Scale (ARS) [22]. The ARS is a behavior-rating scale consisting of 18 items, nine inattention items and nine hyperactive-impulsivity items. The K-ARS parent and teacher forms have been shown to be valid and reliable [23].

\section{Blood sample collection and testing}

For ADHD patients and healthy controls, blood samples were drawn from the participants' antecubital veins between 08:00 and 12:00 hour. Blood samples $(2 \mathrm{ml})$ were collected in a lithium heparin vacuum tube. After centrifugation (3,000 rpm, 10 minutes), plasma samples were divided into microcentrifuge tubes and frozen immediately at $-70^{\circ} \mathrm{C}$ until use.

\section{Plasma levels of EPO}

For all patients and healthy controls, blood samples were drawn from the antecubital veins of subjects between 7 and 8 a.m. following an overnight fast. Approximately $10 \mathrm{ml}$ of blood was collected and placed in lithium heparin vacuum tubes. Blood Plasma separated by centrifuge and stored at -80 until analysis. EPO levels were 
assayed using the sandwich ELISA method (BMS2035; eBioscience, Vienna, Austria) following the manufacturer's instructions. The optical density of the color reaction in the wells was read using a microtiter plate reader ( $\mu$ Quant; BioTek, Winooski, VT, USA) set for $450 \mathrm{~nm}$. Duplicate readings were averaged for each standard, control, and sample, subtracting the average zero standard optical density. For the standard curve, the data points were linearized by plotting the log of the EPO concentration vs. the log of the optical density, and the best fit line was determined by regression analysis. The EPO level of each sample was determined by the concentration read from the standard curve. The intra- and inter-assay coefficients of variance were less than $5 \%$. All assays for both patients and controls were carried out in the same run in duplicate by the same operator.

\section{Data Analyses}

We performed all statistical analyses using STATA (version 11.0; StataCorp, College Station, TX, USA). Comparison tests between ADHD patients and normal controls for continuous and categorical variables were conducted using the $t$ test and chi-squared test, as appropriate. Unadjusted difference in the plasma EPO levels between the two groups was assessed using the Wilcoxon rank-sum test be-

Table 1. Demographic and psychometric variables in the study subjects

\begin{tabular}{lcc}
\hline \multicolumn{1}{c}{ Variable } & $\begin{array}{c}\text { Normal controls } \\
(\mathrm{n}=81)\end{array}$ & $\begin{array}{c}\text { ADHD } \\
(\mathrm{n}=78)\end{array}$ \\
\hline Sex* (male/female) & $31 / 50$ & $64 / 14$ \\
Age (yr), (range) & $9.1 \pm 1.4(7-14)$ & $8.6 \pm 2.2(6-14)$ \\
KEDI-WISC & & \\
$\quad$ Verbal IQ & & $103.1 \pm 12.4$ \\
$\quad$ Performance IQ & & $101.6 \pm 13.9$ \\
$\quad$ Total IQ & & $102.7 \pm 11.6$ \\
K-ARS subscale score & & \\
$\quad$ Inattention score* & $4.4 \pm 2.0$ & $14.1 \pm 6.1$ \\
$\quad$ Hyperactivity-impulsivity & $3.5 \pm 2.2$ & $12.9 \pm 5.4$ \\
$\quad$ Score* & & \\
$\quad$ Total score* & $7.9 \pm 3.8$ & $27.4 \pm 9.8$ \\
ADS T-score & & \\
$\quad$ Omission error & & $73.1 \pm 24.5$ \\
Commission error & & $76.1 \pm 24.3$ \\
$\quad$ Response time & & $52.9 \pm 16.3$ \\
$\quad$ Response variability & & $83.5 \pm 27.5$ \\
\hline
\end{tabular}

Values are presented as number only or mean \pm standard deviation. ADHD, attention deficit-hyperactivity disorder; KEDI-WISC, Korean version of the Wechsler intelligence scale for children; K-ARS, Korean ADHD rating scale; ADS, computerized ADHD diagnostic system. ${ }^{*} p<0.01$. cause the plasma EPO values were not normally distributed. Correlation coefficients between plasma EPO and K-ARS subscale scores were calculated using spearman's correlation. And we categorized the plasma EPO levels in tertiles, based on the distribution in the whole study subjects. Differences (95\% confidence intervals, 95\% Cls) in each K-ARS subscale scores (Inattention score, Hyperactivity-impulsivity score and Total score) were estimated comparing highest and the second highest tertiles to the lowest tertile (baseline) of the plasma EPO using linear regression (i.e., tertile 2 and 3 vs. tertile 1). We also used logistic regression to examine odds ratios of $\mathrm{ADHD}$ associated with increased plasma EPO levels. A probability level of $p$ less than 0.05 was considered statistically significant.

\section{RESULTS}

\section{Demographic Characteristics and Evaluation of Clinical Symptoms}

The ADHD group consisted of 64 boys and 14 girls, and the healthy control group consisted of 31 boys and 50 girls. The average age of the ADHD group was $8.6 \pm 2.2$ years, and that of the healthy control group was $9.1 \pm 1.4$ years. There was no significant difference in age. There were significant differences in sex $(p<0.01)$ and K-ARS scores $(p<0.01)$ between both groups. Baseline IQ scores and computerized ADHD diagnostic system T-scores for the ADHD group are presented in Table 1.

\section{Comparison of EPO Levels between ADHD Patients and Healthy Controls}

The median plasma EPO level in ADHD patients was 12.9 (interquartile range $[\mathrm{IQR}]=7.9-21.1) \mathrm{mlU} / \mathrm{ml}$, whereas it was $12.0(\mathrm{IQR}=6.5-19.7) \mathrm{mIU} / \mathrm{ml}$ in the healthy controls. This difference was not statistically significant (Wilcoxon rank-sum test; Fig. 1).

\section{Association of EPO Levels and ADHD}

Participants in the highest tertile of plasma EPO had a 1.49 times higher risk of ADHD than those in the lowest tertile $(95 \% \mathrm{Cl}[0.63,3.55])$, and those in the second highest tertile had a 2.39 times higher risk of ADHD than those in the lowest tertile $(95 \% \mathrm{Cl}[0.97,5.94])$, but there were no statistically significant relationships between plasma EPO levels and ADHD after adjusting for age and sex 


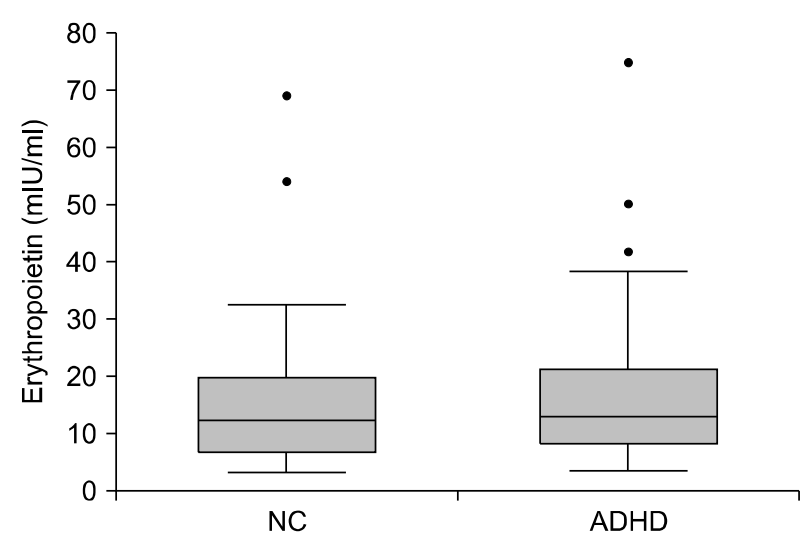

Fig. 1. Plasma levels of EPO in normal controls and ADHD patients. ADHD, attention deficit-hyperactivity disorder; EPO, erythropoietin; NC, normal controls.

Wilcoxon rank-sum test was used for the comparison between two groups.

Table 2. Odds ratios (95\% confidence interval) for ADHD by plasma EPO levels (tertile)*

\begin{tabular}{cccc}
\hline $\begin{array}{c}\text { Plasma EPO } \\
\text { level }(\mathrm{mlU} / \mathrm{ml})\end{array}$ & $\begin{array}{c}\text { Case/ } \\
\text { Non-case }\end{array}$ & Model 1 & Model 2 \\
\hline$<9$ & $23 / 30$ & 1 (reference) & 1 (reference) \\
$9-17.9$ & $25 / 28$ & $1.46(0.68,3.14)$ & $2.39(0.97,5.94)$ \\
$>17.9$ & $26 / 27$ & $1.35(0.63,2.91)$ & $1.49(0.63,3.55)$ \\
\hline
\end{tabular}

EPO, erythropoietin; ADHD, attention deficit-hyperactivity disorder. Tertiles are based on the distribution in the whole study subjects. Model 1 was not adjusted. Model 2 was adjusted for age and sex.

*Logistic regression was used for the test.

(Table 2).

\section{Correlation between EPO Levels and K-ARS Scores in ADHD Patients and Healthy Controls}

Plasma EPO levels correlated positively with some K-ARS scores (including hyperactivity-impulsivity score and total score) as determined by Spearman's correlation test in ADHD patients and in healthy controls $(r=0.157$, $p<0.05$, and $r=0.162, p<0.05$, respectively; Table 3).

Adjusting for age and sex, a linear regression analysis (Model 2) showed that inattention score was significantly higher $(3.04,95 \% \mathrm{Cl}[0.64,5.44])$ in the second highest tertile of plasma EPO compared to those in the lowest tertile. Hyperactivity-impulsivity score was significantly higher $(2.19,95 \% \mathrm{Cl}[0.10,4.38])$ in the highest tertile of plasma EPO compared to those in the lowest tertile. Moreover, total K-ARS score was significantly higher $(5.06,95 \% \mathrm{Cl}[0.74,9.38])$ in the second highest tertile of
Table 3. Correlation between EPO and K-ARS subscale scores in study subjects (Spearman's correlation coefficients)

\begin{tabular}{llll}
\hline \multicolumn{1}{c}{ Variable } & EPO & $\begin{array}{c}\text { Inattention } \\
\text { score }\end{array}$ & $\begin{array}{c}\text { Hyperactivity- } \\
\text { impulsivity } \\
\text { score }\end{array}$ \\
\hline Inattention score & 0.146 & & \\
Hyperactivity-impulsivity score & $0.157^{*}$ & $0.826^{* *}$ & \\
Total score & $0.162^{*}$ & $0.938^{* *}$ & $0.949^{* *}$ \\
\hline
\end{tabular}

EPO, erythropoietin; K-ARS, Korean ADHD rating scale. ${ }^{*} p<0.05,{ }^{* *} p<0.01$.

Table 4. Difference (95\% confidence interval) in K-ARS subscale scores by plasma EPO levels (tertile)*

\begin{tabular}{ccc}
\hline $\begin{array}{c}\text { Plasma EPO } \\
\text { level }(\mathrm{mlU} / \mathrm{ml})\end{array}$ & Model 1 & Model 2 \\
\hline $\begin{array}{c}\text { Inattention score } \\
<9\end{array}$ & 0 (reference) & 0 (reference) \\
$9-17.9$ & $2.06(-0.5,4.61)$ & $3.04(0.64,5.44)$ \\
$>17.9^{*}$ & $1.57(-0.99,4.12)$ & $1.70(-0.67,4.07)$ \\
Hyperactivity-impulsivity score & 0 (reference) \\
$<9$ & 0 (reference) & $2.00(-0.21,4.21)$ \\
$9-17.9$ & $1.26(-1.12,3.685)$ & \\
$>17.9$ & $2.15(-0.23,4.54)$ & $2.19(0.10,4.38)$ \\
Total score & 0 (reference) & 0 (reference) \\
$<9$ & $3.32(-1.35,7.99)$ & $5.06(0.74,9.38)$ \\
$9-17.9$ & $4.02(-0.65,8.69)$ & $4.2(-0.06,8.46)$ \\
$>17.9$ & &
\end{tabular}

Tertiles are based on the distribution in the whole study subjects. Model 1 was not adjusted. Model 2 was adjusted for age and sex. * Linear regression was used for the test.

plasma EPO compared to those in the lowest tertile (Table 4).

\section{DISCUSSION}

In the present study, we investigated plasma EPO levels in children with ADHD and found that plasma EPO levels correlated positively with K-ARS scores, including inattention, hyperactivity-impulsivity and total scores, in ADHD children and healthy controls. Our findings show that a higher peripheral EPO synthesis or release is related to higher inattention score, hyperactivity-impulsivity score, and total K-ARS score in ADHD children and healthy controls.

The present study also found that EPO levels were associated with an impairment in executive functions, including inattention and hyperactivity-impulsivity. It remains unclear whether the alteration in plasma EPO levels rep- 
resents a pathological mechanism or a compensatory mechanism. In our knowledge, this is the first study offering an analysis of plasma EPO levels in children with ADHD. To the best of our knowledge, no previous studies have investigated the relationship between cognitive function in children and EPO levels.

Neonatal hypoxia-ischemia affects motor and cognitive function [24]. ADHD has been related with early hypoxia-ischemia and prematurity [25]. The dopaminergic neurotransmission from the mesencephalon to the forebrain is a key mechanism for proper motor and cognitive processing, and the dopamine uptake transporter blocker, methylphenidate, is effective in alleviating hyperactivity. The pathophysiology underlying ADHD may involve disruption of dopaminergic neurotransmission [26]. EPO has trophic effects on dopaminergic neurons. EPO also stimulates striatal dopamine release [27].

According to a recent study, high endogenous plasma EPO levels have been associated with acute neurologic deficits in Kenyan children with CM [15]. Severe malaria causes upregulation of endogenous EPO levels [28]; levels caused by this upregulation can be greater than plasma levels caused by similar anemia without malaria [29]. An Ugandan study in children with CM also reported that elevated plasma EPO levels are associated with coma prolongation and mortality increase in children with $\mathrm{CM}$ [30]. The biological relevance of plasma EPO is usually difficult to interpret in the absence of any direct measurement of EPO in the brain. EPO is found to permeate the blood brain barrier (BBB) by active translocation, possibly via $E P O$ receptors expressed in the brain vasculature pattern [31].

A study assessing the levels of EPO in serum and cerebrospinal fluid (CSF) of epileptic children reported that EPO is produced locally in the brain in response to neuronal injury due to epileptic seizures [32]. The CSF EPO levels in epileptic children were significantly higher than those of control children because the CSF level of EPO determines the neuroprotective capacity of the brain. The serum EPO levels of refractory epileptic children did not differ significantly from those of control children. From the abovementioned, several conclusions can be drawn: that $E P O$ is produced by the brain in response to neuronal injury due to epileptic seizures, that the CSF EPO level determines the neuroprotective capacity of the brain, that CSF EPO levels can be used as a marker of epileptic seiz- ures, and that CSF EPO levels can predict prognosis and degree of neuronal damage. Nakamura et al. [33] suggest that, although the serum EPO concentrations were not different between depressed patients and healthy controls, the brain of depressed individuals may be hypoxic and that an increased EPO in the CSF may contribute to limit hypoxia-induced damage to neurons in depressed individuals. Consistent with those findings, our present study did not find a statistically significant difference in plasma EPO levels between children with ADHD and healthy controls either.

Serum EPO can only permeate an impaired BBB. Thus, the aforementioned findings suggest that CSF EPO found in patients with normal BBB function is most probably produced in the brain itself [34]. The permeability of EPO might also not be clearly confirmed. Further studies are required to clearly understand the source and role of EPO in ADHD.

The present study has some limitations. First, the relatively small sample size might have limited the generalizability of our results. Second, the origins of plasma EPO remain unclear. Although EPO is principally produced in the kidneys, it is also found in neurons and astrocytes [11]. Furthermore, it is uncertain whether EPO levels in plasma are correlated with EPO levels in the brain. Third, in the present study, we measured only the plasma levels of EPO from blood samples of children and adolescents, resulting in a broad range of results, which was the major limitation of this study. Although, to date, EPO levels were analyzed in patients with a variety of disorders, the results from these studies have been largely inconclusive because of this particular limitation.

Future studies are needed to establish the most reliable, accurate method for measurement of EPO and to determine which source of EPO (platelets, plasma, serum, whole blood, or CSF) provides the most reliable biological marker of ADHD. Although we did not find the difference between ADHD patients and healthy controls, we examined whether there was any correlation between plasma EPO levels and clinical characteristics of ADHD. Further studies on larger samples will help to clarify some important aspects of the pathophysiology of ADHD and the clinical significance of EPO levels (its brain or blood levels) for children with ADHD. 


\section{Acknowledgments}

This work was supported by a grant (2020R1I1A3A040 36435) awarded by the Basic Science Research Program through the National Research Foundation of Korea (NRF) and funded by the Ministry of Education.

This study was also supported by Soonchunhyang University.

\section{- Conflicts of Interest}

No potential conflict of interest relevant to this article was reported.

\section{- Author Contributions}

Authors Se-hoon Shim and Yong-Ku Kim had full access to all study data, and take responsibility for the integrity of the data and accuracy of data analysis. Se-hoon Shim, Yong-Ku Kim, Young Hwangbo, Ji Sun Kim, Hee-jung Yoon, Youn Jung Lee, Young Sup Woo, and Won-Myong Bahk drafted the manuscript. All authors developed the study concept and design, and aided in data interpretation, as well as critical revisions of the manuscript. Se-hoon Shim and Won-Myong Bahk reviewed the manuscript. All authors approved the final version of the paper for submission.

\section{口 ORCID}

Se-hoon Shim https://orcid.org/0000-0002-3137-6591 Yong-Ku Kim https://orcid.org/0000-0001-5694-7840 Young Hwangbo https://orcid.org/0000-0002-8447-8372 Hee-jung Yoon https://orcid.org/0000-0001-9860-4774 Ji Sun Kim https://orcid.org/0000-0003-2472-4591 Youn Jung Lee https://orcid.org/0000-0003-4208-7039 Young Sup Woo https://orcid.org/0000-0002-0961-838X Won-Myong Bahk https://orcid.org/0000-0002-0156-2510

\section{REFERENCES}

1. Nigg JT, Casey BJ. An integrative theory of attention-deficit/ hyperactivity disorder based on the cognitive and affective neurosciences. Dev Psychopathol 2005; 17:785-806.

2. Polanczyk G, de Lima MS, Horta BL, Biederman J, Rohde LA. The worldwide prevalence of ADHD: a systematic review and metaregression analysis. Am J Psychiatr 2007; 164:942-948.

3. Kim JY, Ahn DH, Shin YJ. An epidemiological study of attention-deficits hyperactivity disorder and learning disabilities in a rural area. J Korean Neuropsychiatr Assoc 1999;38:784-793.

4. American Psychiatric Association. Diagnostic and statistical manual of mental disorders: DSM-5. 5th ed. Arlington: American Psychiatric Association;2013.

5. Kelly AM, Margulies DS, Castellanos FX. Recent advances in structural and functional brain imaging studies of attentiondeficit/hyperactivity disorder. Curr Psychiatry Rep 2007;9: 401-407.

6. Maxwell PH, Ferguson DJ, Nicholls LG, Iredale JP, Pugh CW, Johnson MH, et al. Sites of erythropoietin production. Kidney Int 1997;51:393-401.

7. Koury ST, Bondurant MC, Koury MJ, Semenza GL. Localization of cells producing erythropoietin in murine liver by in situ hybridization. Blood 1991;77:2497-2503.

8. Schuster SJ, Koury ST, Bohrer M, Salceda S, Caro J. Cellular sites of extrarenal and renal erythropoietin production in anaemic rats. Br I Haematol 1992;81:153-159.

9. Digicaylioglu M, Bichet S, Marti HH, Wenger RH, Rivas LA, Bauer $\mathrm{C}$, et al. Localization of specific erythropoietin binding sites in defined areas of the mouse brain. Proc Natl Acad Sci U S A 1995;92:3717-3720.

10. Conrad KP, Benyo DF, Westerhausen-Larsen A, Miles TM. Expression of erythropoietin by the human placenta. FASEB J 1996; 10:760-768.

11. Masuda S, Okano M, Yamagishi K, Nagao M, Ueda M, Sasaki R. A novel site of erythropoietin production. Oxygen-dependent production in cultured rat astrocytes. J Biol Chem 1994; 269:19488-19493.

12. Marti HH, Wenger RH, Rivas LA, Straumann U, Digicaylioglu $\mathrm{M}$, Henn $\mathrm{V}$, et al. Erythropoietin gene expression in human, monkey and murine brain. Eur J Neurosci 1996;8:666-676.

13. Malhotra S, Nijhawan S, Rosenbaum DM. Erythropoietin (epoetin) as a protective factor for the brain. Curr Atheroscler Rep 2004;6:301-306.

14. Campana WM, Misasi R, O'Brien JS. Identification of a neurotrophic sequence in erythropoietin. Int J Mol Med 1998;1: 235-241.

15. Casals-Pascual C, Idro R, Gicheru N, Gwer S, Kitsao B, Gitau $\mathrm{E}$, et al. High levels of erythropoietin are associated with protection against neurological sequelae in African children with cerebral malaria. Proc Natl Acad Sci U S A 2008;105:26342639.

16. Russell VA. Neurobiology of animal models of attention-deficit hyperactivity disorder. J Neurosci Methods 2007;161:185-198.

17. Viviani B, Bartesaghi S, Corsini E, Villa P, Ghezzi P, Garau A, et al. Erythropoietin protects primary hippocampal neurons increasing the expression of brain-derived neurotrophic factor. J Neurochem 2005;93:412-421.

18. Shim SH, Hwangbo Y, Kwon YJ, Jeong HY, Lee BH, Lee HJ, et al. Increased levels of plasma brain-derived neurotrophic factor (BDNF) in children with attention deficit-hyperactivity disorder (ADHD). Prog Neuropsychopharmacol Biol Psychiatry 2008;32:1824-1828.

19. Yazici KU, Yazici IP, Ustundag B. Increased serum hepcidin levels in children and adolescents with attention deficit hy- 
peractivity disorder. Clin Psychopharmacol Neurosci 2019; 17:105-112.

20. Kim YS, Cheon KA, Kim BN, Chang SA, Yoo HJ, Kim JW, et al. The reliability and validity of Kiddie-Schedule for Affective Disorders and Schizophrenia-Present and Lifetime VersionKorean version (K-SADS-PL-K). Yonsei Med J 2004;45:81-89.

21. Park KS, Yoon JR, Park HJ, Park HJ, Kwon KO. Korean educational development institute-Wechsler intelligence scale for children (KEDI-WISC). Seoul:Korean Educational Development Institute;2002.

22. Barkley RA, Murphy KR. Attention-deficit hyperactivity disorder: a clinical workbook. New York:Guilford Press; 1991.

23. So YK, Noh JS, Kim YS, Ko SG, Koh YJ. The reliability and validity of Korean parent and teacher ADHD Rating Scale. J Korean Neuropsychiatr Assoc 2002;41:283-289.

24. Anderson P, Doyle LW; Victorian Infant Collaborative Study Group. Neurobehavioral outcomes of school-age children born extremely low birth weight or very preterm in the 1990 s. JAMA 2003;289:3264-3272.

25. Lindström K, Lagerroos P, Gillberg C, Fernell E. Teenage outcome after being born at term with moderate neonatal encephalopathy. Pediatr Neurol 2006;35:268-274.

26. Mehler-Wex C, Riederer P, Gerlach M. Dopaminergic dysbalance in distinct basal ganglia neurocircuits: implications for the pathophysiology of Parkinson's disease, schizophrenia and attention deficit hyperactivity disorder. Neurotox Res 2006; 10:167-179

27. Yamamoto $M$, Koshimura $K$, Kawaguchi $M$, Sohmiya $M$, Murakami Y, Kato Y. Stimulating effect of erythropoietin on the release of dopamine and acetylcholine from the rat brain slice. Neurosci Lett 2000;292:131-133.

28. Díez-Padrisa N, Aguilar R, Machevo S, Morais L, Nhampossa T, O'Callaghan-Gordo C, et al. Erythropoietin levels are not independently associated with malaria-attributable severe disease in Mozambican children. PLoS One 2011;6:e24090.

29. Casals-Pascual C, Kai O, Cheung JO, Williams S, Lowe B, Nyanoti $\mathrm{M}$, et al. Suppression of erythropoiesis in malarial anemia is associated with hemozoin in vitro and in vivo. Blood 2006; 108:2569-2577.

30. Shabani E, Opoka RO, Idro R, Schmidt R, Park GS, Bangirana $\mathrm{P}$, et al. High plasma erythropoietin levels are associated with prolonged coma duration and increased mortality in children with cerebral malaria. Clin Infect Dis 2015;60:27-35.

31. Brines ML, Ghezzi P, Keenan S, Agnello D, de Lanerolle NC, Cerami $\mathrm{C}$, et al. Erythropoietin crosses the blood-brain barrier to protect against experimental brain injury. Proc Natl Acad Sci U S A 2000;97:10526-10531.

32. Mahmoud AT, El Deghady AAM. Erythropoietin in the serum and cerebrospinal fluid of epileptic children. Alex J Pediatr 2005; 19:185-192.

33. Nakamura T, Ebihara I, Shimada N, Koide H. Elevated levels of erythropoietin in cerebrospinal fluid of depressed patients. Am J Med Sci 1998;315:199-201.

34. Marti HH, Gassmann M, Wenger RH, Kvietikova I, MorgantiKossmann MC, Kossmann T, et al. Detection of erythropoietin in human liquor: intrinsic erythropoietin production in the brain. Kidney Int 1997;51:416-418. 